OPEN ACCESS

Edited by:

Wei Fan,

Yunnan Agricultural University, China

Reviewed by:

He Qiang Lou,

Zhejiang Agriculture and Forestry

University, China

Dezhi Wu,

Zhejiang University, China

Miguel A. Pineros,

United States Department of

Agriculture (USDA), United States

${ }^{*}$ Correspondence:

Jixing Xia

xiajx@gxu.edu.cn

Zhenhua Ming

zhming@gxu.edu.cn

tThese authors have contributed equally to this work.

Specialty section: This article was submitted to

Plant Abiotic Stress,

a section of the journal

Frontiers in Plant Science

Received: 23 December 2017

Accepted: 17 April 2018

Published: 07 May 2018

Citation:

Lu M, Yang G, Li P, Wang Z, Fu S,

Zhang $X$, Chen $X$, Shi M, Ming Z and

Xia J (2018) Bioinformatic

and Functional Analysis of a Key Determinant Underlying the Substrate Selectivity of the Al Transporter, Nrat1.

Front. Plant Sci. 9:606.

doi: 10.3389/fpls.2018.00606

\section{Bioinformatic and Functional Analysis of a Key Determinant Underlying the Substrate Selectivity of the Al Transporter, Nrat1}

\author{
Muxue Lu+, Guangzhe Yang ${ }^{\dagger}$, Peifang Lit, Zhigang Wang, Shan Fu, Xiang Zhang, \\ Xi Chen, Mingxing Shi, Zhenhua Ming* and Jixing Xia*
}

State Key Laboratory of Conservation and Utilization of Subtropical Agro-Bioresources, College of Life Science and Technology, Guangxi University, Nanning, China

Nrat1 is a member of the natural resistance-associated macrophage protein (Nramp) family of metal ion transporters in all organisms. Different from other Nramp members capable of transporting divalent metals, Nrat1 specifically transports trivalent aluminum (Al) ion. However, molecular mechanism underlying the Al transport selectivity of Nrat1 remains unknown. Here, we performed structure-function analyses of Nrat1 and other Nramp members to gain insights into the determinants of ion selectivity. A phylogenetic analysis showed that plant Nramp transporters could be divided into five groups. OsNrat1 was found in one of the individual clades and clustered with SbNrat1 and ZmNrat1 on the evolutionary tree. Structural modeling revealed that Nrat1 transporters adopted a common LeuT fold shared by many Nramp-family transporters that likely employed an identical transport mechanism. Sequence alignment and evolutionary conservation analysis of amino acids identified a metal-permeation pathway of Nrat1 centered at the metal binding site. The metal binding site of Nrat1 was characterized by two conserved sequence motifs, i.e., the Asp-Pro-Ser-Asn motif (motif A) and the AlaIle-lle-Thr motif (motif B). Replacement of the Ala-Met-Val-Met motif B of the OsNramp3 manganese $(\mathrm{Mn})$ transporter to that of Nrat1 resulted in a partial gain of Al transport activity and a total loss of $\mathrm{Mn}$ in yeast. Conversely, substitution of the motif B of OsNrat1 with that of OsNramp3 altered the Al transport activity. These observations indicated the metal binding site, particularly the motif B, as a key determinant of Al selectivity of Nrat1.

Keywords: Nrat1, aluminum, Al transporter, selectivity, bioinformatic analysis

\section{INTRODUCTION}

The natural resistance-associated macrophage proteins (Nramps) are widely presented in bacteria, fungi, plants, and mammals (Curie et al., 2000; Nevo and Nelson, 2006). They function as metal ion transporters for a wide range of divalent metal substrates such as $\mathrm{Fe}^{2+}, \mathrm{Mn}^{2+}, \mathrm{Cd}^{2+}, \mathrm{Zn}^{2+}, \mathrm{Co}^{2+}$, $\mathrm{Ca}^{2+}, \mathrm{Cu}^{2+}, \mathrm{Ni}^{2+}$, and $\mathrm{Pb}^{2+}$ (Gunshin et al., 1997). In higher plants, Nramp proteins play major roles in the transport of mineral elements from soil to different organs and tissues of plants. For example, AtNramp1 is found to be localized at the plasma membrane of root cells and functions as a high-affinity transporter for Mn uptake in Arabidopsis (Cailliatte et al., 2010). AtNramp3 and 
AtNramp4 function redundantly to release Fe and $\mathrm{Mn}$ from the vacuole (Thomine et al., 2000; Lanquar et al., 2005, 2010). In rice, OsNramp1 transports Fe and Cd in yeast and is suggested to be involved in Cd uptake (Takahashi et al., 2011). OsNramp3 is localized at the plasma membrane of node cells and is involved in distribution of Mn, but not Fe and Cd (Yamaji et al., 2013). The plasma membrane-localized transporter OsNramp5 is the major contributor for Mn and Cd uptake (Sasaki et al., 2012).

Recently, OsNrat1, an Nramp member, was reported to specifically transport $\mathrm{Al}^{3+}$ but not divalent metal ions such as $\mathrm{Fe}^{2+}, \mathrm{Mn}^{2+}$, and $\mathrm{Cd}^{2+}$, and required for $\mathrm{Al}$ tolerance in rice (Xia et al., 2010). In sorghum, SbNrat1, a close homolog of rice OsNrat1, also was shown to selectively transport $\mathrm{Al}^{3+}$ (Lu et al., 2017). However, the molecular mechanisms underlying the $\mathrm{Al}$ transport selectivity of Nrat1 remain unknown.

Several studies have investigated the relationships between the structure and the function in Nramp proteins. For instance, mutational analysis of the first external loop (Loop I) of NRAMP2/DCT1/DMT1 suggested that Loop I is involved in metal ion binding and specificity (Cohen et al., 2003). The mutation (G185R) in NRAMP2/DCT1/DMT1 not only resulted in a decrease in iron transport but increased the permeability to calcium (Xu et al., 2004). In Arabidopsis, three residues (L67, E401, F413) of AtNramp4 have been also shown to play important roles in metal selectivity (Pottier et al., 2015). On the other hand, the crystal structural studies have revealed that Nramp proteins shared a conserved protein fold that was previously found in the amino acid transporter LeuT (Cellier, 2012; Ehrnstorfer et al., 2014). The ScaNramp structure also revealed that a metal binding site consists of conserved aspartate, asparagines, and methionine residues, and a backbone carbonyl from transmembrane segments (TMs) 1 and 6 (Ehrnstorfer et al., 2014). Moreover, the conserved metal-binding site methionine was shown to confer selectivity against the abundant alkaline earth metals calcium and magnesium (Bozzi et al., 2016a). However, the role of the conserved metal-binding site in controlling substrate selectivity is still poorly understood.

In this study, we compared the structure and function of Nratl and other initially reported Nramp members in plants by phylogenetic analysis and homology modeling. Furthermore, we performed the site-direct mutagenesis analysis of the conserved metal binding motif in two Nramp proteins, OsNrat1 and OsNramp3, which are known as transporters for $\mathrm{Al}$ and $\mathrm{Mn}$ (Xia et al., 2010; Yamaji et al., 2013), respectively, and examined their transport activities for $\mathrm{Al}$ and $\mathrm{Mn}$. Our results identified a key determinant of Al selectivity of Nrat1, which is essential for Mn selectivity of OsNramp3. It provides novel insights into the molecular basis of Al transport selectivity of Nrat1 and valuable clues to investigate Mn transport selectivity of OsNramp3.

\section{MATERIALS AND METHODS}

\section{Sequence and Structure Collection}

The amino acid sequences of OsNratl homologs from four types of plants, Oryza sativa, Arabidopsis thaliana, Sorghum bicolor, and Zea mays, were obtained by BLAST
(Johnson et al., 2008) using the OsNrat1 sequence as a query in the U.S. National Center for Biotechnology Information (NCBI) reference sequence (RefSeq) database. After eliminating the repetitive sequences, we collected a total of 24 sequences. The structures of prokaryotic Nramp transporters were downloaded from the Protein Data Bank (PDB) database. The 25 Nramp transporters and their NCBI accession numbers are as follows: OsNramp1, XP_015647629; OsNramp2, XP_015632573; OsNramp3, XP_015644306; OsNrat1, XP_015625418; OsNramp5, XP_015645014; OsNramp6, XP_015620405; OsNramp7, XP_015618209; AtNramp1, NP_178198; AtNramp2, NP_175157; AtNramp3, NP_179896; AtNramp4, NP_201534; AtNramp5, NP_193614; AtNramp6, NP_173048; SbNramp1, XP_002459640; SbNramp2, XP_002465667; SbNramp3, XP_002438846; SbNramp4, XP_021317241; SbNramp5, XP_002461772; SbNramp6, XP_002464246; SbNrat1, XP_002451480; ZmNramp1, XP_008670084; ZmNramp4, XP_008670762; ZmNramp5, XP_008652227; ZmNramp6, XP_008665146; ZmNrat1, NP_001334019.

\section{Sequence Alignment and Phylogenetic Analysis}

Multiple-sequence alignment (MSA) was performed by using the T-Coffee server (Di Tommaso et al., 2011). The alignment was produced by combining multiple methods, including mafft_msa, clustalw_msa and t_coffee_msa. Results were subjected to figure production by ESPript version 3.0 (Robert and Gouet, 2014), evolutionary tree building by MEGA6 (Tamura et al., 2013), or evolutionary conservation analysis by ConSurf (Ashkenazy et al., 2010).

Phylogenetic analysis was conducted in MEGA version 6 by the bootstrap neighbor joining method (Saitou and Nei, 1987). Bootstrap method (Felsenstein, 1985) was used for test of phylogeny and the number of bootstrap replications was set to 1000 . The evolutionary distances were calculated using the Poisson correction method (Zuckerkandl and Pauling, 1965) and were in the units of the number of amino acid substitutions per site. The analysis involved all 25 amino acid sequences of the Nramp family transporters in the four types of plants. All positions containing gaps and missing data were eliminated. There was a total of 450 positions in the final dataset.

\section{Evolutionary Conservation Analysis}

MSA of the 25 plant Nramp transporters constructed by T-Coffee and the I-TASSER model for the core domain of OsNrat1 (45-502) was used to calculate the position-specific conservation scores by the empirical Bayesian algorithms (Mayrose et al., 2004) in ConSurf (Ashkenazy et al., 2010). The continuous conservation scores are divided into a discrete scale of nine grades for visualization, from the most variable positions (grade 1) colored turquoise, through intermediately conserved positions (grade 5) colored white, to the most conserved positions (grade 9) colored maroon. Scripts for visualizing the protein colored with ConSurf scores were generated and the colored protein was shown in PyMOL (DeLano, 2002). 
Normalized conservation scores were also extracted and used to calculate the average conservation score for each structural element and produce figures in GraphPad Prim version 5.

\section{Vector Construction}

The coding region of OsNrat1 and OsNramp3 was amplified from rice (Oryza sativa, Nipponbare) root cDNA with high-fidelity PCR (KOD Fx polymerase, Toyobo), and the amplified fragments were cloned into the HindIII/EcoRI, BamHI/EcoRI restriction sites of yeast expression vector pYES2 (Invitrogen), respectively. Site-directed mutagenesis of OsNrat1 and OsNramp3 was performed by overlapping PCR (Ho et al., 1989). The wild-type and mutated OsNrat1 or OsNramp3 CDS were verified by sequencing. All the PCR primers used are listed in Supplementary Table S1.

\section{Yeast Assays}

The yeast strains used in this study were BY4741 (MATa his $2 \Delta 0$ met15 $\Delta 0$ ura3 $\Delta 0$ ) and smf1 (MATa his $2 \Delta 0$ met15 $\Delta 0$ ura3 $\triangle 0$ YOL122c::KanMX4). Al sensitivity test on agar and complementation of the $s m f 1$ phenotype were performed as described by Xia et al. (2010). For Al sensitivity evaluation, OsNrat1, OsNramp3, mutated OsNrat1, or OsNramp3, and vector control pYES2 were introduced into yeast strain BY4741 and then spotted on solid media (LPM with $2 \%$ galactose for induction of the GAL promoter) containing 0,200 , or $300 \mu \mathrm{M}$ $\mathrm{AlCl}_{3}$ buffered with $5 \mathrm{mM}$ succinic acid. For $\mathrm{Al}$ uptake in liquid culture, transformants were selected on uracil-deficient medium and grown in synthetic complete (SC-uracil) yeast solution containing $2 \%$ glucose. Cells at mid-exponential phase were harvested and transferred to LPM medium containing 2\% galactose. Cells were cultured for $2 \mathrm{~h}$. Then $\mathrm{AlCl}_{3}$ was added to the cell culture at the final concentration of $50 \mu \mathrm{M} \mathrm{AlCl}$. After $6 \mathrm{~h}$ incubation with shaking, cells were harvested by centrifugation at $12000 \times g$ for $5 \mathrm{~min}$, and washed three times with deionized water (MilliQ; Millipore), dried and then digested with $65 \% \mathrm{HNO}_{3}$. Al concentration was measured by inductively coupled plasma optical emission spectrometry.

\section{RESULTS}

\section{Plant Nramp Transporters Fall Into Five Groups on the Phylogenetic Tree}

The amino acid sequence of OsNrat1 was used to retrieve Nramp homologs in four plant species by BLAST (Johnson et al., 2008). Twenty-four protein sequences were selected for phylogenetic analysis, including six from Oryza sativa (OsNrat1, OsNramp13, and 5-7), six from Arabidopsis thaliana (AtNramp1-6), seven from Sorghum bicolor (SbNrat1 and SbNramp1-6), and five from Zea mays (ZmNrat1, ZmNramp1, and ZmNramp46). The resulting phylogenetic tree includes five main clades corresponding to five distinctive Nramp groups (Figure 1). Notably, two known transporters for trivalent Al ion, OsNrat1 (Xia et al., 2010, 2014; Li et al., 2014) and SbNrat1 (Lu et al., 2017), an Nrat1-like transporter of Zea mays (ZmNrat1) as well, are located in the same clade (group III) but separated from other divalent ion transporters on the evolutionary tree.

\section{Nrat1 Transporter Adopts a Conserved LeuT Fold}

Sequence alignment of the 25 plant Nramp transporters and the Staphylococcus Capitis Divalent Metal Ion Transporter (ScaDMT) reveals that they contain a conserved core domain (amino acid sequence 45-502 in OsNrat1, see Supplementary Figure S1). The available structural information on the Nramp family is limited to the prokaryotic homologues of divalent metal transporters. To understand the mechanism in which trivalent $\mathrm{Al}$ is recognized by Nrat1 transporter, we modeled the protein structures of core domain of OsNrat1 and OsNramp3 through an iterative threading algorithm using the I-TASSER server (Roy et al., 2010), as the terminal $\mathrm{N}$ - and $\mathrm{C}$ - regions with unknown functionality are not as important as the core domain that is highly conserved and constitutes a part of the molecular determinants for ion permeation. To compare the architecture of metal binding sites in the ionic binding state of these two proteins, the Mn-binding structure of ScaDMT (PDBID: 5M95), which showed the highest sequence similarities with OsNrat1 (59\%) and OsNramp3 (62\%), was used as a template. The estimated TM-score and C-score of OsNrat1 are $0.95 \pm 0.05$ and 1.68 , respectively; while those of OsNramp3 are $0.92 \pm 0.06$ and 1.50, respectively. Hence, these two models appear to be acceptable.

OsNrat1 adopts a common LeuT fold (Figure 2) that is associated with many prokaryotic Nramp-family transporters, including the Staphylococcus divalent metal transporter ScaDMT (59\% sequence similarity, PDBID: 5M95), the Deinococcus radiodurans Nramp homolog (DraNramp, 56\% similarity, PDBID: 5KTE), and the Eremococcus coleocola Manganese Transporter (EcoDMT, 56\% similarity, PDBID: 5M87). OsNrat1 contains a compact globular domain of 12 transmembrane segments (TMs), of which TMs 1-5 and 6-10 form two inverted repeats of the LeuT fold. Like other LeuT-type transporters, the first TM in each of the two inverted repeats (TM1 and TM6) of OsNrat1 contains two $\alpha$-helices disrupted by a short discontinuous stretch in the middle (Supplementary Figure S1). Overall, a helical bundle comprising TMs 3-5 and 8-10 forms a semicircular (letter $C$ shaped) structure that wraps partway around a second helical bundle formed by TMs 1, 2, 6, and 7 . Substrate transport of the LeuT-type transporters is likely to be coupled with a switch from outward-open to inward-open conformation, through a rigid-body rotation (Shi, 2013) of the moving portion (corresponding to the latter $\alpha$ helical bundle) related to the non-moving portion (corresponding to the former helical bundle).

\section{The Metal Transport Mechanism of Nrat1 Is Conserved}

Evolutionary conservation analysis by the ConSurf server (Ashkenazy et al., 2010) reveals an overwhelming conservation of residues that make up the interior of the Nrat1 cylinder structure (Figure 2A), which contains a substrate transport path along the central axis that is perpendicular to the lipid 

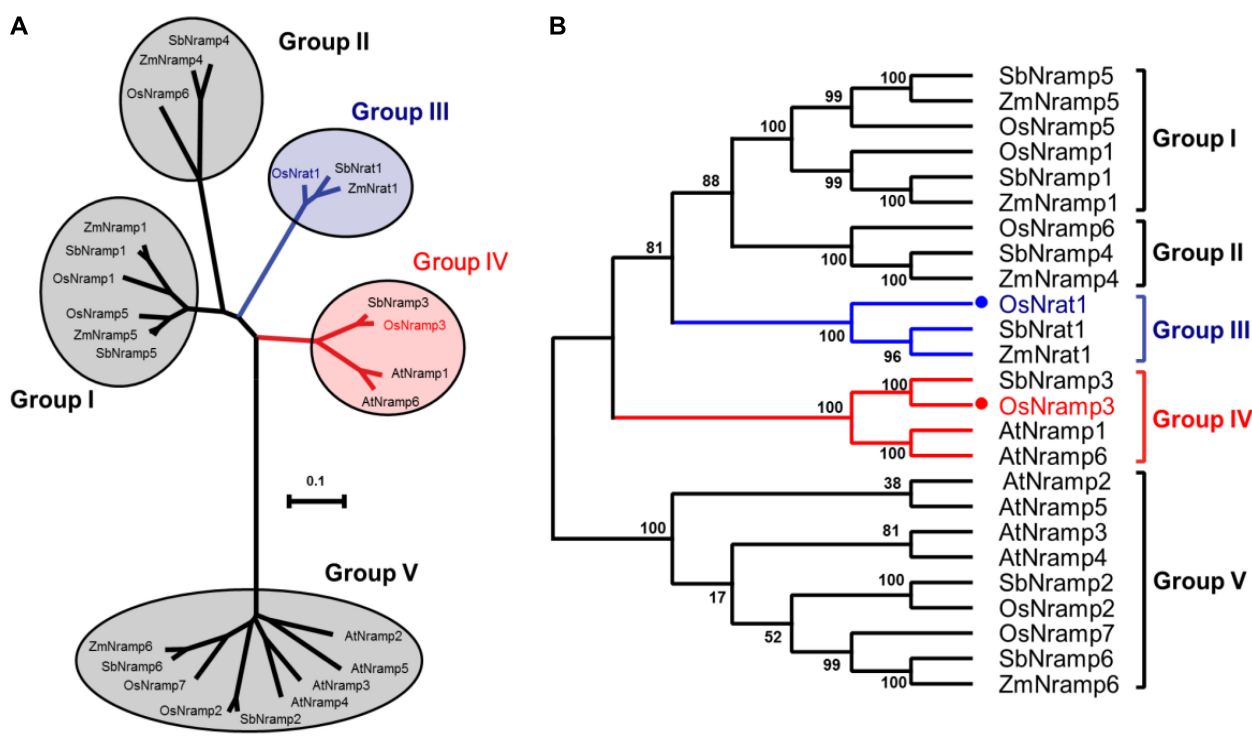

FIGURE 1 | Evolutionary relationships of the plant Nramp family transporters. (A) An unrooted phylogenetic tree for the 25 plant metal transporters of the Nramp family was built by MEGA6. The five clades of the evolutionary tree are indicated by circles, with the OsNrat1 containing group (group III) and the OsNramp3 containing group (group IV) colored in blue and red, respectively. The tree is drawn to scale, with branch lengths in the same units as those of the evolutionary distances used to infer the phylogenetic tree. (B) Related to (A), the evolutionary tree is shown in a squarish-corner style. The percentages of replicate trees in which the associated taxa clustered together in the bootstrap test (1000 replicates) are shown next to the branches.

membrane plane. A detailed conservation analysis for each structural element was performed by calculating the normalized evolutionary conservation scores on all amino acid residues. As shown in Figure 2B, TMs 1-3, 6, 8-10, along with the L23 loop are highly conserved across all 25 Nramp transporters in plants, suggesting that these conserved elements may play important roles in metal transport. On the contrary, structural elements of NT, CT, TM11 and TM12 in the Nramp family are variable. These observations are consistent with structural and functional analysis of other LeuT-type transporters, indicating a conserved substrate transport mechanism. Similar to other known LeuTtype transporters, the five TMs (TMs 1, 3, 6, 8, and 10) may participate directly in substrate binding and transport. TM2 and TM9, which connect the functional helices TM1/TM3 and TM8/TM10, respectively, may confer transport activity through control of the local conformation. The L23 linker that connects neighboring helices of TM2 and TM3 may parallel the roles of TM2 and TM9.

\section{The Nrat1 Transporter Contains a Unique lon Binding Site}

The metal recognition site of prokaryotic Nramp transporters is known to be characterized by two structural motifs (Figure 3 and Supplementary Figure S1), motifs A and B. The highly conserved Asp-Pro-[Gly/Ser]-Asn motif (motif A) occurs in the loop between TM1a and TM1b, as well as the N-terminal portion of TM1b; while the moderate conserved motif (motif B) occurs in the C-terminal portion of TM6a and the loop between TM6a and TM6b. Only four residues, i.e., the first and fourth residues in each motif, are required to coordinate the central metal ion (Figure 3D). The Asp and Asn residues in motif A (locating at the first and fourth position, respectively), as well as the fourth residue in motif $\mathrm{B}$, use their side chains to contact the metal ion. By contrast, the first residue in motif $\mathrm{B}$ contributes to metal binding by its main-chain carbonyl oxygen. In support of the structural and functional importance of the metal coordination ligands, the Asp and Asn residues in motif $\mathrm{A}$ are invariant within the Nramp family and across plant species (Figure 3A and Supplementary Figure S1). Notably, the metal ligands in motif $\mathrm{B}$ are only moderately conserved (Figure 3A and Supplementary Figure S1), suggesting that this motif may contribute to ion-subtype specificity. It is also worth noting that, through refining the proper conformation of terminal residues in each motif, interspace residues of the two motifs may be important for ion binding and selectivity as well.

Sequence alignment results for the two signature motifs show good agreement with the phylogenetic analysis of plant Nramp transporters (Figure 3A). Transporters of the Nrat1 group and group II have an Asp-Pro-Ser-Asn sequence pattern of motif A, while other groups consist of a motif A with a uniform Asp-ProGly-Asn sequence. The characteristic sequence patterns of motif $\mathrm{B}$ can be clearly divided into five sets, each corresponding to one of the five phylogenetic groups. Among the five sets of motif B, sequences of groups I, III, and IV have the highest conservation. Motif B of group III transporters has an invariant sequence of Ala-Ile-Ile-Thr, while motif B of group I and IV has an identical sequence of Ala-Leu-Val-Met and Ala-Met-Val-Met, respectively. Together, these data indicated that Nrat1 transporters contain a unique pair of sequence motifs which may be critical for mediating metal recognition. 

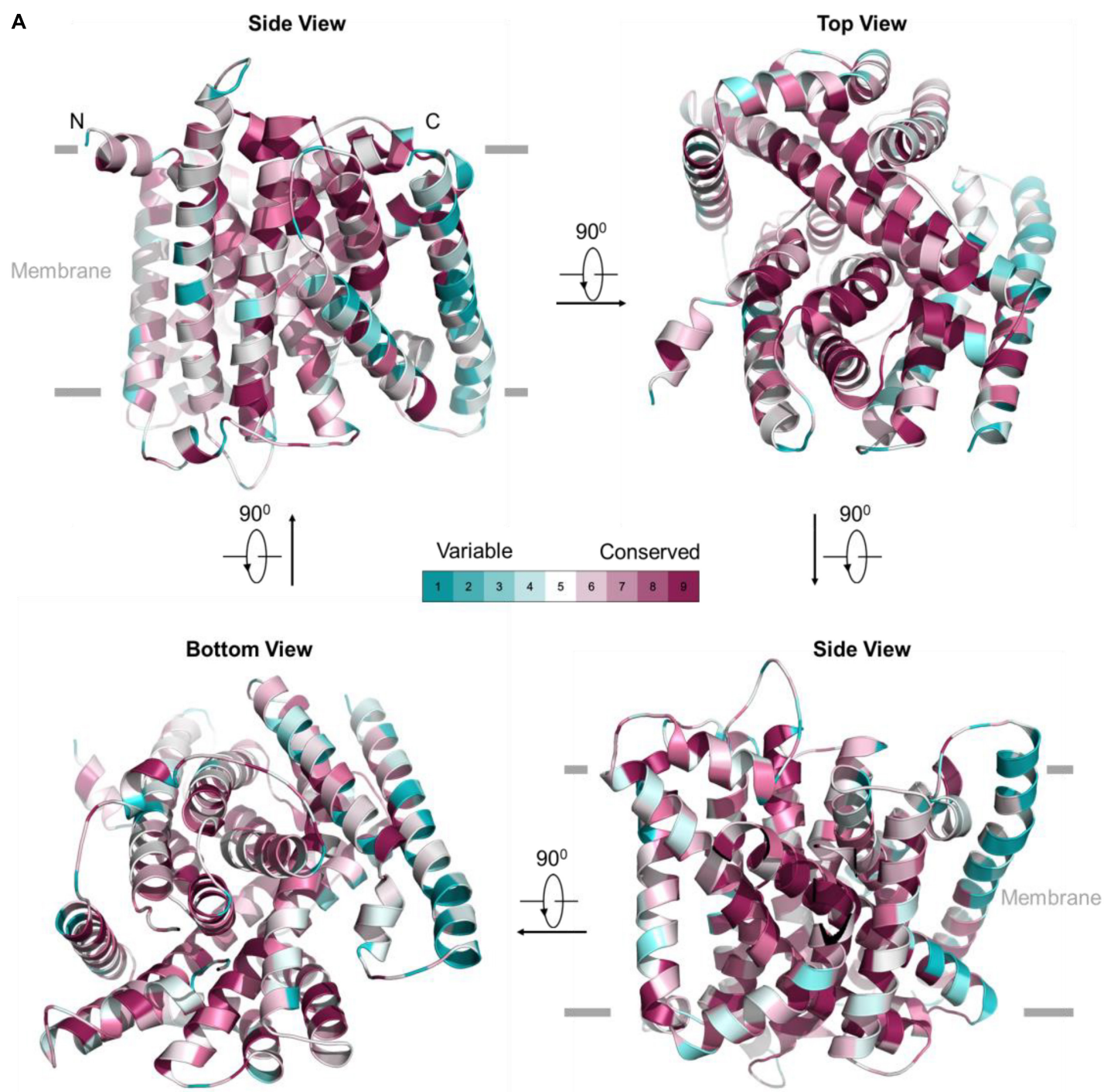

B

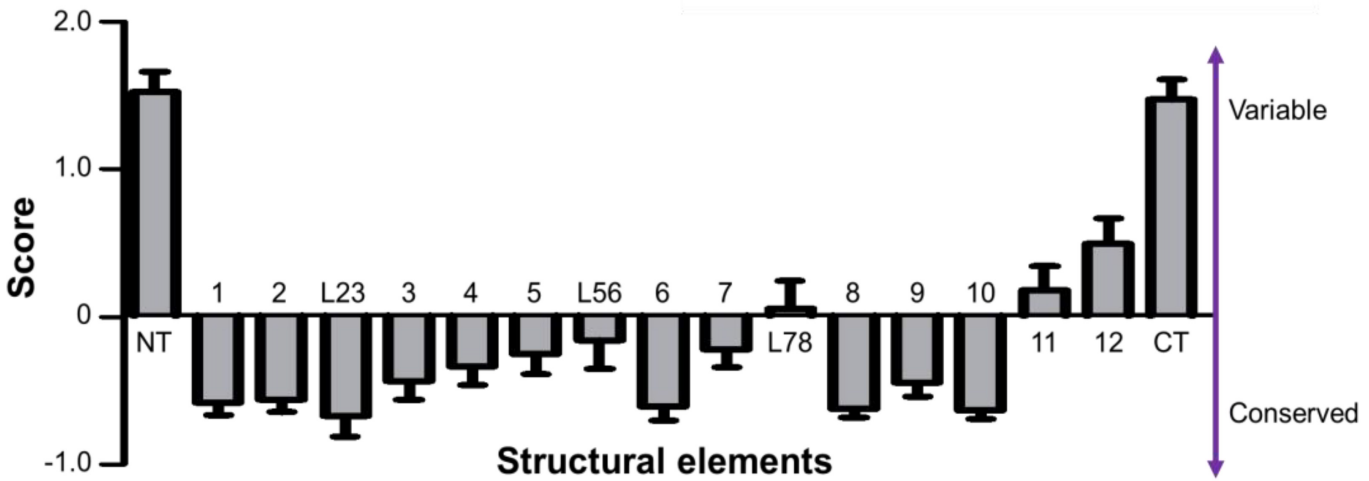

FIGURE 2 | Evolutionary conservation analysis for OsNrat1. The 25 plant Nramp family transporters were used to perform evolutionary conservation analysis by ConSurf. (A) Mapping of evolutionary conservation scales for amino acid positions in the core structure of OsNrat1 (45-502). Residues are colored by their conservation grades (1-9) using the color-coding bar, from turquoise to maroon indicating variable to conserved. The structure is shown in four orientations in cartoon representation. (B) Normalized evolutionary conservation score for each structural element of OsNrat1. The lowest score indicates that this position is the most conserved in this specific protein calculated using a specific multiple sequence alignment (MSA). Error bars represent SEMs. The NT and CT regions of OsNrat1 are not shown in (A). 
A

\begin{tabular}{ll} 
& \\
A & \\
ZmNramp5 & \\
ZmNramp5 \\
OsNramp5 \\
OsNramp1 \\
SbNramp1 \\
ZmNramp1 \\
OsNramp6 \\
SbNramp4 \\
ZmNramp4 \\
\hline OsNrat1 \\
SbNrat1 \\
ZmNrat1 \\
SbNramp3 \\
\hline OsNramp3 \\
AtNramp1 \\
AtNramp6 \\
AtNramp5 \\
AtNramp3 \\
AtNramp4 \\
AtNramp2 \\
SbNramp2 \\
OsNramp2 \\
OsNramp7 \\
SbNramp6 \\
ZmNramp6 \\
ScaDMT
\end{tabular}

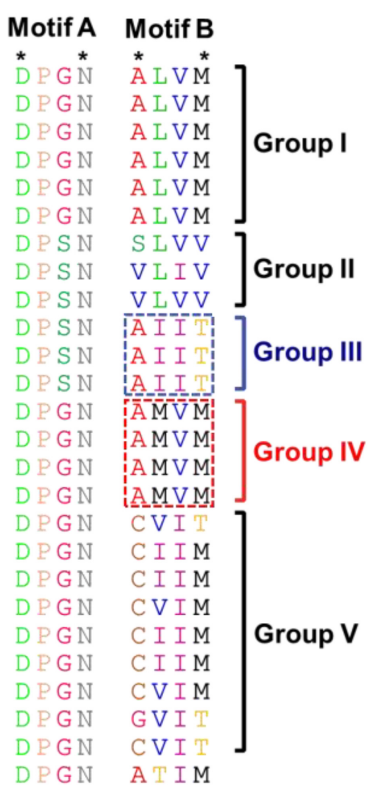

B
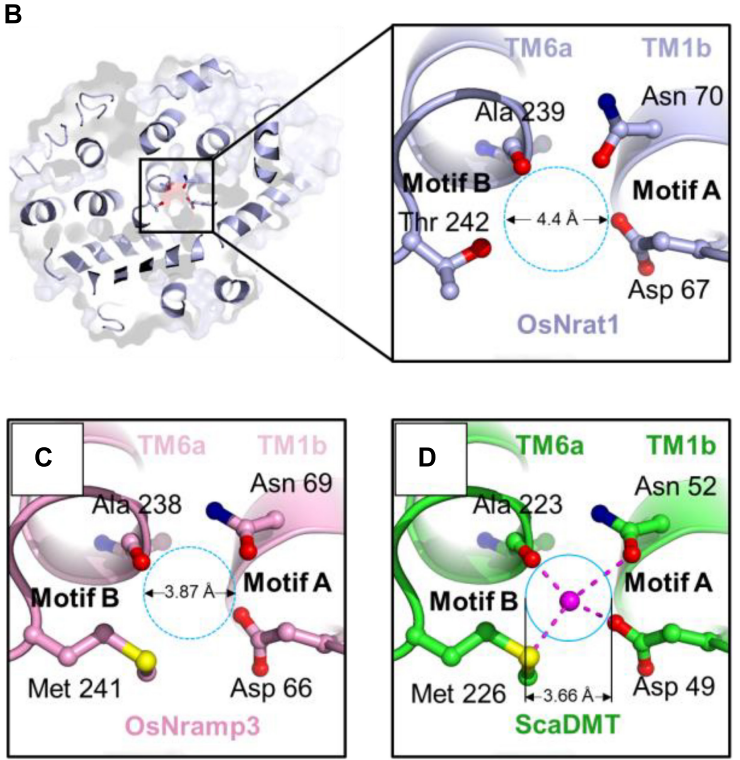

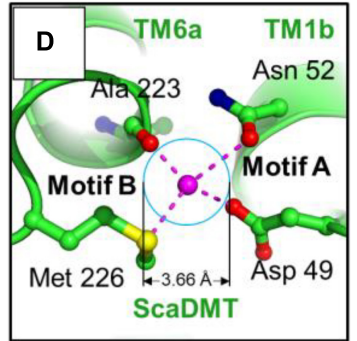

FIGURE 3 | OsNrat1 has a unique metal binding site. (A) Sequence alignment of the two signature motifs of the 25 plant Nramp transporters and a bacteria Nramp transporter ScaDMT. The variations of amino acids in the two motifs were marked with different colors. The four residues involved in metal coordination are indicated as stars above the alignment. OsNrat1, OsNramp3, and ScaDMT are indicated by arrows in light blue, pink and green, respectively. The five phylogenetic groups are labeled to the right of the alignment. The amino acids being swapped in Nrat1 and OsNramp3 are marked by dotted rectangles. (B) A top view of OsNrat1 from the extracellular side of the membrane (Left) with a cartoon representation. The four metal binding residues are shown as colored sticks. The carbon, oxygen, and nitrogen atoms are colored in light blue, red, and tv_blue, respectively. A close-up view of the metal binding site is given in the right panel. (C) A close-up view of the metal binding site of OsNramp3 shown with the same orientation as OsNrat1. The coloring code for the atoms are the same as that in (B), except for carbon (pink) and sulfur (yellow). (D) A close-up view of the binding site of ScaDMT shown with the same orientation as OsNrat1. The coloring pattern for the atoms is the same as that in (C), except for carbon colored green. The proposed metal binding sites of OsNrat1 and OsNramp3 are indicated by dotted circles, while the metal binding site of ScaDMT is indicated by a solid circle. The approximate diameters of the binding sites are calculated by the equation: $D 1=D 2 * d 1 / d 2$. D1 is the diameter of the binding site circle to be calculated. D2 is the spatial distance between the main-chain oxygen of Ala223 and the side-chain oxygen of Asn52 measured in the crystal structure of ScaDMT by the software PyMOL. $d 1$ is the diameter of the binding site circle measured in the figure. $d 2$ is the distance between the main-chain oxygen of Ala223 and the side-chain oxygen of Asn52 of ScaDMT measured in the figure.

We proceeded to compar the putative metal binding sites from the core domain structural models of OsNrat1 and OsNramp3, with that of the crystal structure of ScaDMT. As shown in Figures 3B-D, the architecture of the divalent ion recognition site in OsNramp3 is identical to that observed in ScaDMT. By contrast, the trivalent metal binding site in OsNrat1 appears to be slightly larger than those in OsNramp3 and ScaDMT, as calculated in the two models and the ScaDMT crystal structure (Figures 3B-D). These differences are likely caused by a replacement of the Met with a Thr, which contains a shorter side-chain compared to that of a Met, at the fourth residue of motif B.

\section{The Nrat1 Specific Motif B Is a Key Determinant for Al Transport}

To determine the functional importance of the signature motifs, we generated several mutations for OsNratl and OsNramp3 (OsNrat1 ${ }^{\mathrm{I} 240 \mathrm{M}, \mathrm{I} 241 \mathrm{~V}, \mathrm{~T} 242 \mathrm{M} \text {, OsNrat1 }}{ }^{\mathrm{T} 242 \mathrm{M}}$, OsNramp3 $3^{\mathrm{M} 2391, V 240 I, M 241 \mathrm{~T}}$, OsNramp3 $3^{\mathrm{M} 241 \mathrm{~T}}$, see Supplementary Table S2) by exchanging corresponding residues of one protein with another and examined their capabilities on $\mathrm{Al}$ and Mn transport as well as OsNrat1 or OsNramp3 as a positive control, respectively.
In the absence of $\mathrm{Al}$, all the transformants showed similar growth on the plate (Figure 4A). However, in the presence of $\mathrm{Al}$, the growth of yeast cells carrying OsNrat $1^{\mathrm{T} 242 \mathrm{M}}$, OsNramp3 $3^{\mathrm{M} 239 \mathrm{I}, \mathrm{V} 240 \mathrm{I}, \mathrm{M} 241 \mathrm{~T}}$, or OsNrat1 was significantly inhibited compared with that of the vector control, while that of OsNrat $1^{\mathrm{I} 240 \mathrm{M}, \mathrm{I} 241 \mathrm{~V}, \mathrm{~T} 242 \mathrm{M}}$, OsNramp $3^{\mathrm{M} 241 \mathrm{~T}}$, or OsNramp3 was not (Figure 4A). Al uptake also significantly increased in the

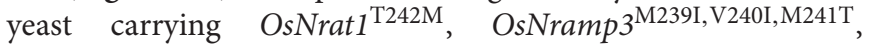
or OsNrat1 and was not affected in the yeast carrying

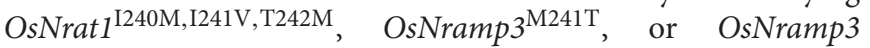
(Figure 4B). Furthermore, the $\mathrm{Al}$ uptake ability of OsNrat $1^{\mathrm{T} 242 \mathrm{M}}$ or OsNramp3 $3^{\mathrm{M} 2391, \mathrm{~V} 2401, \mathrm{M} 241 \mathrm{~T}}$ was lower than that of OsNrat1 (Figure 4B). These results suggested that substitution of the intact motif $B$ of OsNrat1 (OsNrat1 ${ }^{\mathrm{I} 240 \mathrm{M}, \mathrm{I} 241 \mathrm{~V}, \mathrm{~T} 242 \mathrm{M})}$ ) with that of OsNramp3 completely deprived the $\mathrm{Al}$ transport activity of OsNrat1, while a single mutation on the fourth residue Thr242Met $\left(\mathrm{OsNrat} 1^{\mathrm{T} 242 \mathrm{M}}\right.$ ) resulted in a decrease in $\mathrm{Al}$ uptake of OsNrat1, and that replacement of the intact motif $\mathrm{B}$ of OsNramp3 (OsNramp3 ${ }^{\mathrm{M} 2391, \mathrm{~V} 2401, \mathrm{M} 241 \mathrm{~T}}$ ) with that of OsNrat1, but not a single mutation on the fourth residue Met241Thr (OsNramp3 ${ }^{\mathrm{M} 241 \mathrm{~T}}$ ), rendered the $\mathrm{Mn}$ specific divalent transporter to gain a function of $\mathrm{Al}$ transport. 


A
pYES2
OsNrat1
OsNramp3
OsNrat1 ${ }^{\text {I240M, 1241V, T242M }}$
OsNrat1 ${ }^{\text {T242M }}$
OsNramp3 ${ }^{\text {M2391, V2401, M241T }}$
OsNramp3 ${ }^{\text {M241T }}$

$\mathrm{Al}(\mu \mathrm{M})$

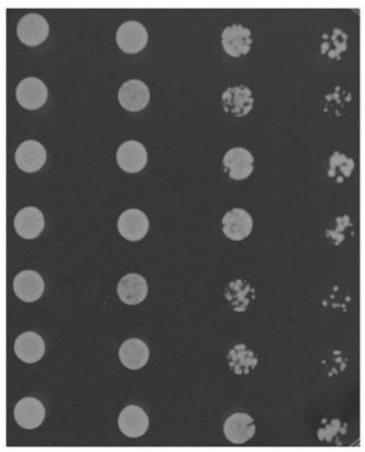

0

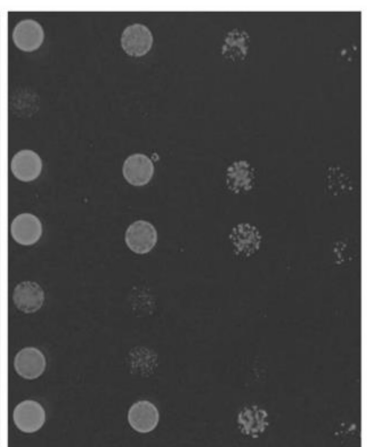

200

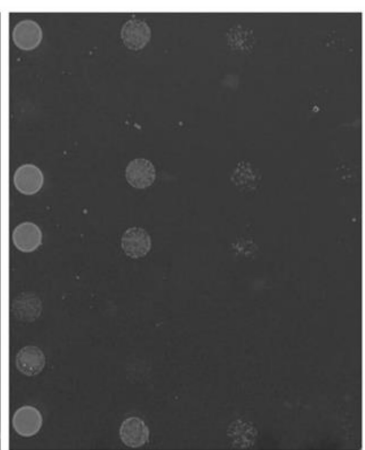

300

B

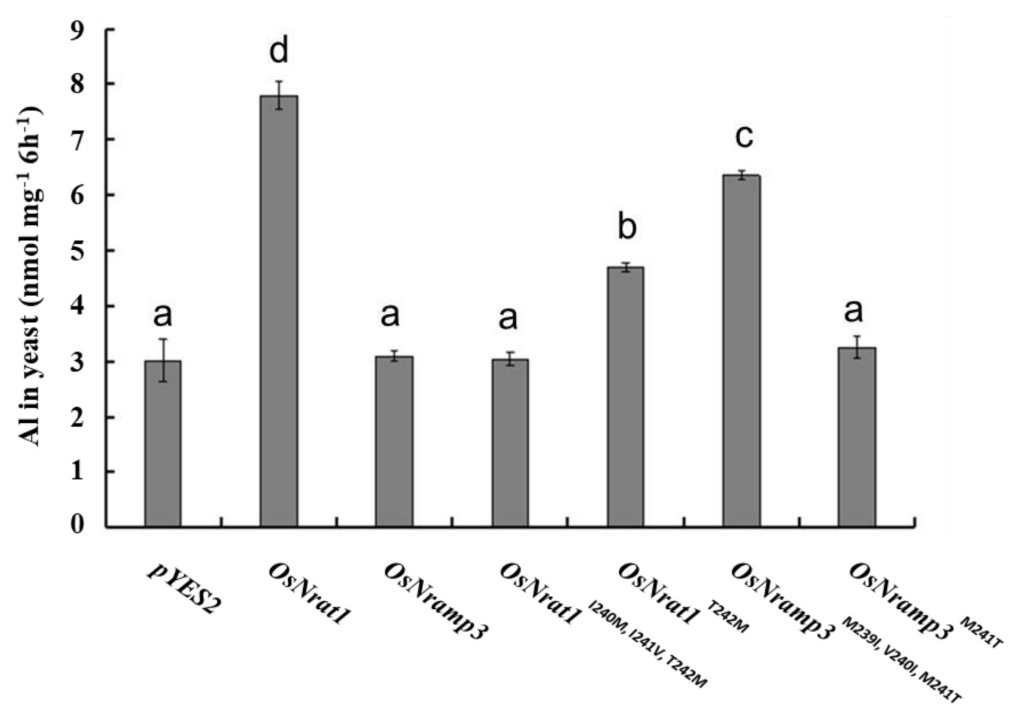

C

pYES2

OsNrat1

OsNramp3

OsNrat1 ${ }^{1240 \mathrm{M}, 1241 \mathrm{~V}, \mathrm{~T} 242 \mathrm{M}}$

OsNrat1 ${ }^{\mathrm{T} 242 \mathrm{M}}$

OsNramp3

M2391, V240I, M241T

OsNramp3 $3^{\text {M241T }}$

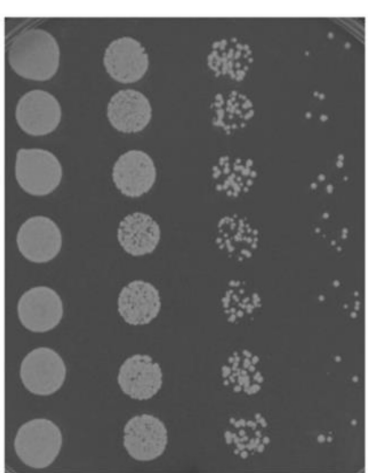

0

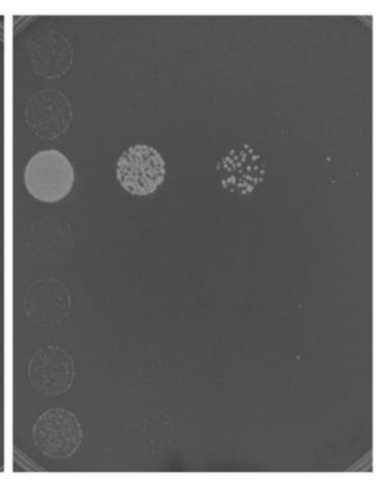

FIGURE 4 | Influence of the signature motif substitution of OsNrat1 or OsNramp3 on transport activity for Al and Mn. (A) Effect of mutated OsNrat1 or OsNramp3 on Al tolerance. Yeast strain (BY4741) transformed with empty vector pYES2, OsNrat1, OsNramp3, OsNrat1 1240M,1241V,T242M , OsNrat1 T242M,

OsNramp3 ${ }^{\mathrm{M} 2391, \mathrm{~V} 240 \mathrm{I}, \mathrm{M} 241 \mathrm{~T}}$, OsNramp3 ${ }^{\mathrm{M} 241 \mathrm{~T}}$ were spotted on LPM without uracil medium (pH 4.2) buffered with 5 mM succinic acid with or without AlCl 3 at serial dilutions (from left to right: $10 \mu \mathrm{l}$ cell suspension with OD 0.2, 0.02, 0.002, and 0.0002) and incubated at $30^{\circ} \mathrm{C}$ for 3 days. (B) Transport activity of mutated OsNrat1 or OsNramp3 for Al. Yeast cells expressing different mutants were exposed to a solution containing $50 \mu \mathrm{M} \mathrm{AlCl} 3$ (pH 4.2) for $6 \mathrm{~h}$. Data are mean $\pm \mathrm{SD}$ of three biological replicates. Different letters above the bars indicate significant differences $(P<0.05$, Tukey's test). (C) Complementation of manganese uptake.

Transformed smf1 were grown on a medium (pH 6.0) buffered with $50 \mathrm{mM} \mathrm{MES}$ in the presence or absence of EGTA. The plates were incubated at $30^{\circ} \mathrm{C}$ for 3 days. 
Subsequently, we performed a complementation test in the $\Delta s m f 1$ yeast strain to examine whether mutants of OsNrat1 and OsNramp3 could have transport activity for Mn. As expected, OsNramp3 could restore the growth of a yeast mutant (smf1) defective in Mn uptake, while OsNratl could not (Figure 4C). Surprisingly, as shown in Figure 4C, all of the four above mentioned mutations failed to complement the manganese uptake phenotype of the $\Delta s m f 1$ mutant yeast. These observations collectively indicate that the Nratl specific motif B is both sufficient and required for $\mathrm{Al}$ transport, while the OsNramp3 specific motif $B$, especially the fourth ionic coordination ligand, is only required but not sufficient for Mn transport.

We also studied the functional importance of residues in close vicinity of the signature motifs by sequence exchange between OsNrat1 and OsNramp3 (OsNrat1 ${ }^{\mathrm{A} 59 \mathrm{~F}, \mathrm{G} 64 \mathrm{~A}}$, OsNrat1 ${ }^{\mathrm{Y} 244 \mathrm{H}}$, OsNramp3 $3^{\text {F58A, A63G }}$, OsNramp3 $3^{\mathrm{H} 243 \mathrm{Y}}$, see Supplementary Table S2). As shown in the Supplementary Figure S2, the expression of OsNrat $1^{\mathrm{A} 59 \mathrm{~F}, \mathrm{G} 64 \mathrm{~A}}$, OsNrat $1^{\mathrm{Y} 244 \mathrm{H}}$, or OsNrat1 increased the sensitivity of yeast to $\mathrm{Al}$ toxicity and the Al uptake in yeast compared with that of the vector control, while that of OsNramp $3^{\mathrm{F} 58 \mathrm{~A}, \mathrm{~A} 63 \mathrm{G}}$, OsNramp $3^{\mathrm{H} 243 \mathrm{Y}}$, or OsNramp3 did not (Supplementary Figures S2A,B). Furthermore, the Al uptake ability of OsNrat $1^{\mathrm{A} 59 \mathrm{~F}, \mathrm{G} 64 \mathrm{~A}}$ or OsNrat $1^{\mathrm{Y} 244 \mathrm{H}}$ was lower than that of OsNrat1 (Supplementary Figure S2B). On the other hand,

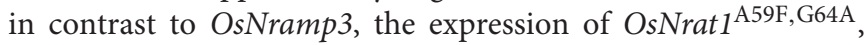
OsNrat $1^{\mathrm{Y} 244 \mathrm{H}}, \mathrm{OsNramp}^{\mathrm{F} 58 \mathrm{~A}, \mathrm{~A} 63 \mathrm{G}}$, or OsNramp3 $3^{\mathrm{H} 243 \mathrm{Y}}$ was not able to complement the growth of the yeast mutant $\Delta s m f 1$ under the Mn-limited condition controlling by EGTA (Supplementary Figure S2C). These results indicated that flanking residues of the characteristic motifs are dispensable for Al selectivity but required, at least in part, for $\mathrm{Al}$ transport activity. These data also suggested that residues near the metal binding motifs of OsNramp3, but not OsNrat1, are essential for Mn uptake of the transporter.

\section{DISCUSSION}

A number of reported variations in the Nrat1 coding region affect transport activity (Li et al., 2014; Xia et al., 2014; Lu et al., 2017) but not selectivity of Nrat1. We reasoned that careful examination of the metal binding site may facilitate to understand the selectivity of the transporter. The Nramp family of transporters utilizes two separate motifs, each from one of the two discontinuous TMs, to coordinate metal ions (Ehrnstorfer et al., 2014, 2017; Bozzi et al., 2016b). Our bioinformatic and functional analyses demonstrate that the metal binding site, particularly the motif B with a sequence of Ala-Ile-Ile-Thr, is a prominent determinant of Al selectivity for Nrat1.

Motif B of OsNramp3 is probably essential for the selectivity of the transporter. However, the interpretation for selectivity of Nrat1 cannot be directly applied to give a simplified explanation for the selectivity of the Mn specific transporters of the Nramp family, as substitution of mere motif B in OsNrat1 by that of OsNramp3 is not sufficient for the former to gain Mn transport activity. This is consistent with the experimental observations for divalent Nramp transporters reported by Bozzi et al. (2016a).
The conserved metal-binding methionine (Met230) of motif B is dispensable in the bacterial DraNramp, as the Met-to-Ala mutant can still enable robust transport of the physiological manganese substrate and similar divalent iron and cobalt. In sharp contrast to the DraNramp, the corresponding Met265Ala mutant of human Nramp2 did not transport any of the tested divalent metals, including $\mathrm{Co}, \mathrm{Mn}, \mathrm{Cd}$, and $\mathrm{Ca}$. These results indicate a dependency of the functional divergence on sequence and structure context (Bozzi et al., 2016a). Supporting this hypothesis, whereas the single mutation (corresponding to Gln76 of OsNrat1) in TM1b of the mammalian transporter DCT1 (Slc11a2) completely blocked Mn transport, a double mutation (corresponding to Asp74 and Gln76 of OsNrat1) in TM1b restored the activity and altered the metal ion specificity in favor of Fe (Cohen et al., 2003). Moreover, random mutagenesis studies revealed that three residues, Leu67 (in the immediate vicinity of motif A) from TM1a and Glu401/Phe413 from TM10, contributed to the selectivity of AtNramp4 for the uptake of another divalent metal Cd (Pottier et al., 2015).

Our work identified that the Nrat1-type motif B is both sufficient and required for Al transport in Nrat1 and OsNramp3, as one of the key determinants for the Al selectivity. Our results also suggested that the OsNramp3-type motif B is necessary, though not sufficient, for the Mn selectivity of OsNramp3. Identification of the important functions of motif B in substrate selectivity of Nrat1 and OsNramp3 may help further elucidate the selectivity of other Nramp transporters.

\section{AUTHOR CONTRIBUTIONS}

ZM and JX conceived and designed the experiments. ML, GY, PL, $\mathrm{ZW}, \mathrm{XZ}, \mathrm{XC}, \mathrm{SF}$, and MS performed the experiments. ML, GY, $\mathrm{ZM}$, and JX analyzed the data. ZM and JX wrote the paper.

\section{FUNDING}

This work was supported by Guangxi Natural Science Foundation (2015GXNSFCB139001, 2016GXNSFFA380013, 2016GXNSFBA380176), National Natural Science Foundation of China (31670253, 31700052), the Project of High Level Innovation Team and Outstanding Scholar in Guangxi Colleges and Universities (2016), and China Postdoctoral Science Foundation (2017M612863).

\section{ACKNOWLEDGMENTS}

We thank Zheng Zhang (Shandong University) for helpful discussion.

\section{SUPPLEMENTARY MATERIAL}

The Supplementary Material for this article can be found online at: https://www.frontiersin.org/articles/10.3389/fpls.2018.00606/ full\#supplementary-material 


\section{REFERENCES}

Ashkenazy, H., Erez, E., Martz, E., Pupko, T., and Ben-Tal, N. (2010). ConSurf 2010: calculating evolutionary conservation in sequence and structure of proteins and nucleic acids. Nucleic Acids Res. 38, W529-W533. doi: 10.1093/nar/g kq399

Bozzi, A. T., Bane, L. B., Weihofen, W. A., Mccabe, A. L., Singharoy, A., Chipot, C. J., et al. (2016a). Conserved methionine dictates substrate preference in Nramp-family divalent metal transporters. Proc. Natl. Acad. Sci. U.S.A. 113, 10310-10315. doi: 10.1073/pnas.1607734113

Bozzi, A. T., Bane, L. B., Weihofen, W. A., Singharoy, A., Guillen, E. R., Ploegh, H. L., et al. (2016b). Crystal structure and conformational change mechanism of a bacterial nramp-family divalent metal transporter. Structure 24, 2102-2114. doi: 10.1016/j.str.2016.09.017

Cailliatte, R., Schikora, A., Briat, J. F., Mari, S., and Curie, C. (2010). Highaffinity manganese uptake by the metal transporter NRAMP1 is essential for Arabidopsis growth in low manganese conditions. Plant Cell 22, 904-917. doi: $10.1105 /$ tpc. 109.073023

Cellier, M. F. (2012). Nramp: from sequence to structure and mechanism of divalent metal import. Curr. Top. Membr. 69, 249-293. doi: 10.1016/B978-012-394390-3.00010-0

Cohen, A., Nevo, Y., and Nelson, N. (2003). The first external loop of the metal ion transporter DCT1 is involved in metal ion binding and specificity. Proc. Natl. Acad. Sci. U.S.A. 100, 10694-10699. doi: 10.1073/pnas.1934572100

Curie, C., Alonso, J. M., Le Jean, M., Ecker, J. R., and Briat, J. F. (2000). Involvement of NRAMP1 from Arabidopsis thaliana in iron transport. Biochem. J. 347(Pt 3), 749-755. doi: 10.1042/bj3470749

DeLano, W. (2002). The PyMOL Molecular Graphics System. San Carlos, CA: Delano Scientific.

Di Tommaso, P., Moretti, S., Xenarios, I., Orobitg, M., Montanyola, A., Chang, J. M., et al. (2011). T-Coffee: a web server for the multiple sequence alignment of protein and RNA sequences using structural information and homology extension. Nucleic Acids Res. 39, W13-W17. doi: 10.1093/nar/gkr245

Ehrnstorfer, I. A., Geertsma, E. R., Pardon, E., Steyaert, J., and Dutzler, R. (2014). Crystal structure of a SLC11 (NRAMP) transporter reveals the basis for transition-metal ion transport. Nat. Struct. Mol. Biol. 21, 990-996. doi: $10.1038 /$ nsmb.2904

Ehrnstorfer, I. A., Manatschal, C., Arnold, F. M., Laederach, J., and Dutzler, R. (2017). Structural and mechanistic basis of proton-coupled metal ion transport in the SLC11/NRAMP family. Nat. Commun. 8:14033. doi: 10.1038/ ncomms 14033

Felsenstein, J. (1985). Confidence limits on phylogenies: an approach using the bootstrap. Evolution 39, 783-791. doi: 10.1111/j.1558-5646.1985.tb00420.x

Gunshin, H., Mackenzie, B., Berger, U. V., Gunshin, Y., Romero, M. F., Boron, W. F., et al. (1997). Cloning and characterization of a mammalian protoncoupled metal-ion transporter. Nature 388, 482-488. doi: 10.1038/41343

Ho, S. N., Hunt, H. D., Horton, R. M., Pullen, J. K., and Pease, L. R. (1989). Sitedirected mutagenesis by overlap extension using the polymerase chain reaction. Gene 77, 51-59. doi: 10.1016/0378-1119(89)90358-2

Johnson, M., Zaretskaya, I., Raytselis, Y., Merezhuk, Y., Mcginnis, S., and Madden, T. L. (2008). NCBI BLAST: a better web interface. Nucleic Acids Res. 36 W5-W9. doi: 10.1093/nar/gkn201

Lanquar, V., Lelievre, F., Bolte, S., Hames, C., Alcon, C., Neumann, D., et al. (2005) Mobilization of vacuolar iron by AtNRAMP3 and AtNRAMP4 is essential for seed germination on low iron. EMBO J. 24, 4041-4051. doi: 10.1038/sj.emboj. 7600864

Lanquar, V., Ramos, M. S., Lelievre, F., Barbier-Brygoo, H., Krieger-Liszkay, A., Kramer, U., et al. (2010). Export of vacuolar manganese by AtNRAMP3 and AtNRAMP4 is required for optimal photosynthesis and growth under manganese deficiency. Plant Physiol. 152, 1986-1999. doi: 10.1104/pp.109. 150946

Li, J. Y., Liu, J., Dong, D., Jia, X., Mccouch, S. R., and Kochian, L. V. (2014). Natural variation underlies alterations in Nramp aluminum transporter (NRAT1) expression and function that play a key role in rice aluminum tolerance. Proc. Natl. Acad. Sci. U.S.A. 111, 6503-6508. doi: 10.1073/pnas.1318975111
Lu, M., Wang, Z., Fu, S., Yang, G., Shi, M., Lu, Y., et al. (2017). Functional characterization of the SbNratl gene in sorghum. Plant Sci. 262, 18-23. doi: 10.1016/j.plantsci.2017.05.010

Mayrose, I., Graur, D., Ben-Tal, N., and Pupko, T. (2004). Comparison of sitespecific rate-inference methods for protein sequences: empirical Bayesian methods are superior. Mol. Biol. Evol. 21, 1781-1791. doi: 10.1093/molbev/ msh194

Nevo, Y., and Nelson, N. (2006). The NRAMP family of metal-ion transporters. Biochim. Biophys. Acta 1763, 609-620. doi: 10.1016/j.bbamcr.2006.05.007

Pottier, M., Oomen, R., Picco, C., Giraudat, J., Scholz-Starke, J., Richaud, P., et al. (2015). Identification of mutations allowing Natural Resistance Associated Macrophage Proteins (NRAMP) to discriminate against cadmium. Plant J. 83, 625-637. doi: 10.1111/tpj.12914

Robert, X., and Gouet, P. (2014). Deciphering key features in protein structures with the new ENDscript server. Nucleic Acids Res. 42, W320-W324. doi: 10. 1093/nar/gku316

Roy, A., Kucukural, A., and Zhang, Y. (2010). I-TASSER: a unified platform for automated protein structure and function prediction. Nat. Protoc. 5, 725-738. doi: 10.1038/nprot.2010.5

Saitou, N., and Nei, M. (1987). The neighbor-joining method: a new method for reconstructing phylogenetic trees. Mol. Biol. Evol. 4, 406-425.

Sasaki, A., Yamaji, N., Yokosho, K., and Ma, J. F. (2012). Nramp5 is a major transporter responsible for manganese and cadmium uptake in rice. Plant Cell 24, 2155-2167. doi: 10.1105/tpc.112.096925

Shi, Y. (2013). Common folds and transport mechanisms of secondary active transporters. Annu. Rev. Biophys. 42, 51-72. doi: 10.1146/annurev-biophys083012-130429

Takahashi, R., Ishimaru, Y., Senoura, T., Shimo, H., Ishikawa, S., Arao, T., et al. (2011). The OsNRAMP1 iron transporter is involved in Cd accumulation in rice. J. Exp. Bot. 62, 4843-4850. doi: 10.1093/jxb/err136

Tamura, K., Stecher, G., Peterson, D., Filipski, A., and Kumar, S. (2013). MEGA6: molecular evolutionary genetics analysis version 6.0. Mol. Biol. Evol. 30, 2725-2729. doi: 10.1093/molbev/mst197

Thomine, S., Wang, R., Ward, J. M., Crawford, N. M., and Schroeder, J. I. (2000) Cadmium and iron transport by members of a plant metal transporter family in Arabidopsis with homology to Nramp genes. Proc. Natl. Acad. Sci. U.S.A. 97, 4991-4996. doi: 10.1073/pnas.97.9.4991

Xia, J., Yamaji, N., Che, J., Shen, R. F., and Ma, J. F. (2014). Differential expression of Nrat 1 is responsible for Al-tolerance QTL on chromosome 2 in rice. J. Exp. Bot. 65, 4297-4304. doi: 10.1093/jxb/eru201

Xia, J., Yamaji, N., Kasai, T., and Ma, J. F. (2010). Plasma membranelocalized transporter for aluminum in rice. Proc. Natl. Acad. Sci. U.S.A. 107, 18381-18385. doi: 10.1073/pnas.1004949107

Xu, H., Jin, J., Defelice, L. J., Andrews, N. C., and Clapham, D. E. (2004) A spontaneous, recurrent mutation in divalent metal transporter-1 exposes a calcium entry pathway. PLoS Biol. 2:E50. doi: 10.1371/journal.pbio.0020050

Yamaji, N., Sasaki, A., Xia, J. X., Yokosho, K., and Ma, J. F. (2013). A node-based switch for preferential distribution of manganese in rice. Nat. Commun. 4:2442. doi: $10.1038 /$ ncomms 3442

Zuckerkandl, E., and Pauling, L. (1965). "Evolutionary divergence and convergence in proteins," in Evolving Genes and Proteins, eds V. Bryson and H. J. Vogel (New York, NY: Academic Press), 97-166. doi: 10.1016/B978-1-4832-2734-4. 50017-6

Conflict of Interest Statement: The authors declare that the research was conducted in the absence of any commercial or financial relationships that could be construed as a potential conflict of interest.

Copyright (C) $2018 \mathrm{Lu}$, Yang, Li, Wang, Fu, Zhang, Chen, Shi, Ming and Xia. This is an open-access article distributed under the terms of the Creative Commons Attribution License (CC BY). The use, distribution or reproduction in other forums is permitted, provided the original author(s) and the copyright owner are credited and that the original publication in this journal is cited, in accordance with accepted academic practice. No use, distribution or reproduction is permitted which does not comply with these terms. 